\title{
Efficacy and safety of DoceAqualip in a patient with locally advanced cervical cancer: A case report
}

\author{
RAMMOHAN PRASANNA ${ }^{1}$, DEEPAK BUNGER ${ }^{2}$ and MUJTABA A. KHAN ${ }^{2}$ \\ ${ }^{1}$ Consultant Medical Oncologist, Department of Medical Oncology, CBCC-GVN Cancer Center, \\ Tiruchirapalli, Tamil Nadu 620005; ${ }^{2}$ Department of Medical Affairs and Clinical Development, \\ Intas Pharmaceuticals Ltd., Ahmedabad, Gujarat 380059, India
}

Received October 10, 2017; Accepted November 27, 2017

DOI: $10.3892 / \mathrm{mco} .2017 .1519$

\begin{abstract}
The standard of care for locally advanced cervical cancer has been the combination of a taxane with platinum based therapy. Conventional docetaxel is known to cause hypersensitivity reactions, fluid retention and other toxicities due to polysorbate- 80 and ethanol. Corticosteroid premedication prior to docetaxel administration is required to prevent these toxicities, however, toxicities are still observed, sometimes fatal, despite premedication. DoceAqualip, a nanosomal docetaxel lipid suspension, developed with lipids generally regarded as safe (GRAS) by the US Food and Drug Administration, is devoid of polysorbate- 80 and ethanol. DoceAqualip has been demonstrated to be effective and well-tolerated in various cancer types. The authors' report a case of a patient with Stage IIIB cervical cancer who was treated with carboplatin and DoceAqualip (concurrent ChemoRT) and achieved complete response without any serious adverse events.
\end{abstract}

\section{Introduction}

In Indian women, cervical cancer is among the most common malignancies, and is a major cause of mortality (1-3). India has a higher incidence of cervical cancer compared with the developed countries (1). The age-adjusted incidence rate of cervical cancer varies widely among Indian registries, from 4.91 to 23.07/100,000 populations; much higher than the world (7.9/100,000 population). Poor to moderate living standards, lack of proper screening, and a high $(\geq 10 \%$ in women aged $>30$ years) prevalence of human papillomavirus infection could be the potential reasons responsible for the higher incidence in India (4).

Correspondence to: Dr Rammohan Prasanna, Consultant Medical Oncologist, Department of Medical Oncology, CBCC-GVN Cancer Center, Trichy-Chennai Trunk Road, Periyar Nagar, Mambalasalai, Tiruchirapalli, Tamil Nadu 620005, India

E-mail: prasumoh81@gmail.com

Key words: cervical cancer, nanosomal docetaxel lipid suspension, DoceAqualip, carboplatin
The treatment of choice for early-stage cervical cancer is radical surgery, whereas, concurrent chemoradiation is preferred for locally advanced stages. However, the treatment options are very limited for patients with recurrent or metastatic cancers. In women with recurrent or limited metastatic disease, surgery or radiotherapy may be effective in only in few cases $(5,6)$. After the clinical alert from the National Cancer Institute (NCI) in 1999 (7), concurrent chemoradiotherapy has become the standard of treatment for locally advanced cervical cancer (8); it enhances the radiosensitivity and local tumor efficacy of cytotoxic agents, with eradication of micrometastasis and ultimately improves the pelvic control and survival (9).

Chemotherapy with a platinum agent (carboplatin/cisplatin) and a taxane based regimen is considered the standard of care for cervical cancer in terms of longer overall survival (OS) and a better quality of life for the patients $(5,7)$. Furthermore, carboplatin has advantages over cisplatin in terms of a better safety profile (low incidence of neuropathy, nephrotoxicity and emetogenesis) (10).

Docetaxel has dose limiting toxicities of acute hypersensitivity reactions, and fluid retention due to the excipients (polysorbate-80 and ethanol) used in the conventional formulations, which require corticosteroid premedication (11). DoceAqualip, a nanosomal docetaxel lipid suspension (NDLS), was developed with lipids generally recognized as safe (GRAS) by the US Food and Drug Administration (USFDA) and is devoid of polysorbate- 80 and ethanol. DoceAqualip has been shown to be more effective than the conventional docetaxel in the treatment of breast cancer (12) and has demonstrated a promising overall response and safety without the need of corticosteroid in the treatment of breast cancer, advanced gastric adenocarcinoma, ovarian cancer, hormone refractory prostate cancer, and non-small cell lung cancer (11-13).

Here, we report a case of a Stage IIIB cervical cancer patient who was treated with concurrent carboplatin and DoceAqualip chemoradiotherapy.

\section{Case report}

A 52-year-old woman presented with complaints of abnormal vaginal bleeding along with white discharge since five months. Her vaginal examination revealed a hard indurated friable 
growth replacing the cervix. Per rectal examination revealed involvement of bilateral parametrium.

Her past medical history included type 2 diabetes and she was taking combination tablet of glimepiride $1 \mathrm{mg}$ and metformin $500 \mathrm{mg}$ twice daily for 3 years. Histopathological examination of the cervical biopsy specimen revealed moderately differentiated squamous cell carcinoma. The magnetic resonance imaging (MRI) of pelvis revealed a large mass of $55 \times 45 \mathrm{~mm}$ in size in the uterine cervix infiltrating the lower third of the myometrium with enlarged bilateral internal iliac nodes, measuring $1.6 \mathrm{~cm}$ on the left side and $1.4 \mathrm{~cm}$ on the right side (Fig. 1). Based on these findings, the patient was diagnosed with squamous cell carcinoma cervix Stage IIIB, with Eastern Cooperation Oncology Group (ECOG) score of 2 and was planned for concurrent chemoradiotherapy.

The patient was started on chemotherapy with docetaxelcarboplatin regimen on December 26, 2014. Carboplatin and nanosomal docetaxel lipid suspension (NDLS; DoceAqualip) were administered intravenously (IV) at doses corresponding to AUC 5-6 (area under the curve; total dose $450 \mathrm{mg}$ ) and $75 \mathrm{mg} / \mathrm{m}^{2}$ (body surface area of $1.67 \mathrm{~kg} / \mathrm{m}^{2}$ ), respectively for 4 cycles (every 3 weekly cycle). DoceAqualip was given without steroid premedication, at a total dose of $120 \mathrm{mg}$ during the first 2 cycles and the dose was reduced to $100 \mathrm{mg}$ for cycles 3 and 4 as the patient developed Grade II neutropenia after 2 cycles of radiotherapy. Post completion of 2 cycles of DoceAqualip, pegylated filgrastim (6 mg) was administered as secondary prophylaxis $24 \mathrm{~h}$ after DoceAqualip administration to avoid the risk of neutropenia. The patient did not develop any hypersensitivity reactions (HSR) or any other serious adverse event despite not using any corticosteroids as premedication. No abnormal laboratory investigations were reported throughout the course of the treatment.

The patient also received concomitant radiotherapy from December 31, 2014 to March 10, 2015 at a dose of 50 Gy to the pelvis and $6120 \mathrm{cGy}$ to bilateral parametrium plus 2 high dose rate intracavitary (HDR ICA)- 15 Gy to point A (paracervical reference point) (6).

Post chemoradiotherapy, her MRI done on April 20, 2015 did not reveal any evidence of residual mass in the cervix and no evidence of enlarged internal iliac or para aortic nodes (Fig. 2). The response could best be graded as a complete response (CR) as per RECIST 1.1 criteria. Her fluorodeoxyglucose (FDG) positron emission tomography-computed tomography (PET-CT) scan done on October 12, 2015 revealed no active disease. The follow-up FDG PET CT whole body scan done on June 5, 2017 did not show any evidence of metabolically active lesion in the uterine cervix or anywhere else in the body.

Consent was obtained from the patient for the publication of this case report and informed consent document was signed by the patient.

\section{Discussion}

Cervical carcinoma is one of the leading malignancies in Indian women with a higher burden as compared to western countries. In India, it accounts for $16 \%$ of all cancers in urban women and $37 \%$ of the cancers in rural women (3). Concurrent chemoradiation using cisplatin has shown $30-50 \%$ reduction in the risk of death, and is regarded the standard treatment for

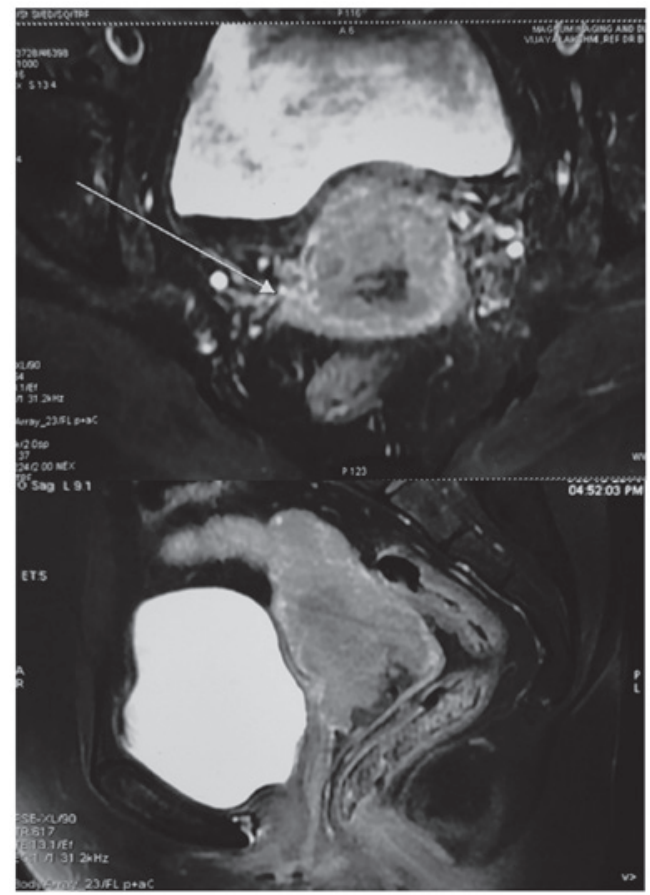

Figure 1. Prechemotherapy MRI pelvis with contrast: Growth in the uterine cervix infiltrating the lower 3rd of myometrium and bilateral parametrium; with enlarged bilateral internal iliac nodes (arrow)

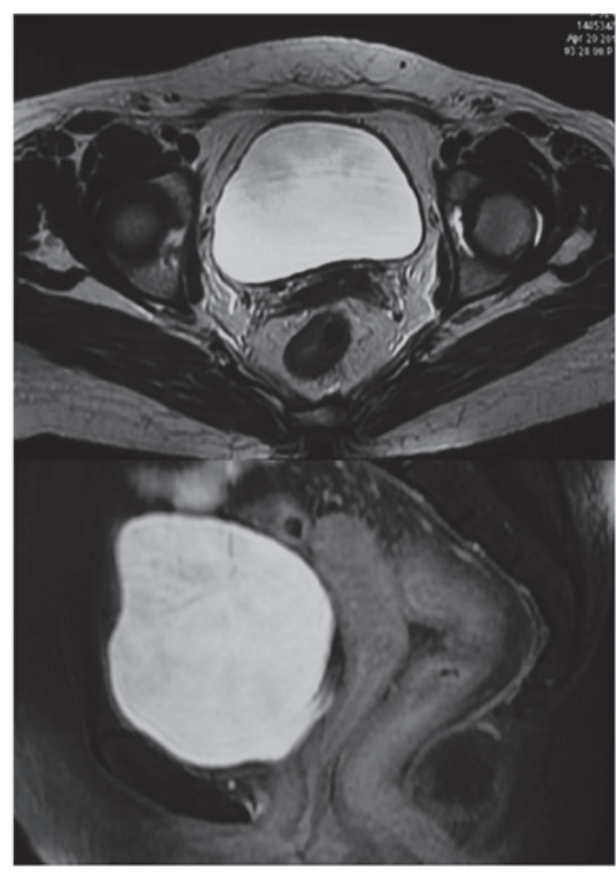

Figure 2. Post-NDLS chemotherapy MRI of abdomen: No evidence of residual mass in the cervix or enlarged internal iliac or paraaortic lymph nodes.

patients with cervical cancer $(7,14,15)$. Carboplatin, an analogue of cisplatin, with a similar mechanism of action, has been used for the treatment of locally advanced cervical carcinoma. It has advantages over cisplatin in terms of decreased nephrotoxicity, neurotoxicity and emetogenesis (16). This favourable toxicity profile when compared to cisplatin may result in better patient adherence to the treatment plan (15). A phase II clinical study 
in patients with recurrent or metastatic squamous carcinoma of the uterine cervix revealed substantially better toxicity profile of carboplatin than cisplatin with similar efficacy (17).

Taxanes are commonly used in the treatment of locally advanced cervical cancer in combination with platinum compounds (18). Docetaxel and carboplatin concurrent chemotherapy has shown to be effective in the treatment of cervical cancer with low incidence of nephrointestinal, neurointestinal, and gastrointestinal toxicities (8). In a phase I study in 20 patients with locally advanced or recurrent cervical cancer, weekly docetaxel and carboplatin (145 cycles) showed a high efficacy in a dose-dense setting. The study demonstrated an overall response rate of $65 \%$, and the treatment was well-tolerated (19).

Similarly, in a pilot study that assessed the efficacy and safety of docetaxel and carboplatin combination therapy in advanced or recurrent uterine cervix cancer in 17 patients yielded $76 \%$ overall response rate (CR in 2 patients, partial response in 11, and stable disease in 4) with no disease progression reported. Overall, it was concluded that combination chemotherapy with docetaxel $\left(60 \mathrm{mg} / \mathrm{m}^{2}\right)$ and carboplatin (AUC 6) was effective and well-tolerated in the treatment of uterine cervix cancer. However, the incidence of Grade 3/4 neutropenia was very high $(76 \%)$, and patients with Grade 4 neutropenia or febrile neutropenia required prophylactic granulocyte colony stimulating factor (G-CSF, $5 \mu \mathrm{g} / \mathrm{kg}$ ) administration (20).

Takekida and colleagues further confirmed the efficacy and safety of docetaxel and carboplatin regimen in the treatment of cervical cancer. In a study of 66 patients (62 treatment naïve and 4 recurrent cancer), docetaxel $\left(60 \mathrm{mg} / \mathrm{m}^{2}\right)$ and carboplatin (AUC 6) combination therapy yielded an overall clinical response rate of $63.7 \%$ (44/66, 95\% confidence interval, 52.1-75.3). Neutropenia was the major haematological toxicity, which rapidly reversed with G-CSF use (8).

Docetaxel is formulated in polysorbate-80 and ethanol, which are known to cause infusion-related toxicities and hypersensitivity reactions, requiring corticosteroid premedication. Thus, a new formulation nanosomal docetaxel lipid suspension (NDLS) was developed using GRAS lipids that is free of polysorbate- 80 and ethanol, and is found to be safe in earlier studies (11-13).

This is the first case report on the use of DoceAqualip in the treatment of cervical cancer. In the current report, the patient who had Stage IIIB cervical cancer, was treated with DoceAqualip and carboplatin combination at generally recommended doses (120 mg followed by $100 \mathrm{mg}$, and AUC 6, respectively) along with radiation therapy, and achieved CR. Overall, the treatment was well-tolerated, even when there was no corticosteroid premedication, hypersensitivity reaction or any other serious adverse event was not reported. Two-year follow-up investigations did not reveal any recurrence of the carcinoma. Ashraf et al, reported that patients receiving DoceAqualip monotherapy did not require G-CSF support whereas dual combination therapy with carboplatin required G-CSF support at each treatment cycle (13). Furthermore, there is a $>20 \%$ risk of febrile neutropenia in patients receiving combination chemotherapy (21). In this case also, the patient developed Grade 2 neutropenia after 2nd dose of radiotherapy; hence, DoceAqualip dose was reduced from 120 to $100 \mathrm{mg}$ after cycle 2 to avoid radiotherapy interruption and further dose delays. Also, after 2 cycles, the patient was given prophylactic G-CSF (pegylated filgrastim) support after each cycle of dual combination therapy with DoceAqualip and carboplatin. Furthermore, it has been established that external-beam and intracavitary radiation along with the concurrent chemotherapy significantly improves survival in locally advanced cervical cancer patients, which was also followed for this patient (6).

The current report highlights the efficacy and safety of DoceAqualip in the treatment of advanced cervical cancer. These results need to be further confirmed in a larger clinical trial.

In conclusion, NDLS (DoceAqualip), a new formulation developed to overcome toxicity and hypersensitivity reactions caused by polysorbate- 80 and ethanol content of the conventional docetaxel formulations, has shown to be effective and well-tolerated in the treatment of cervical cancer without the need for corticosteroid premedication.

\section{Acknowledgements}

We would like to thank Mr. Shreekant Sharma (Lambda Therapeutic Research) for his support in developing the concept/medical writing, additional editorial support and follow-up with the journal/publisher, and Dr Venugopal Madhusudhana (Lambda Therapeutic Research) for additional editorial assistance. Dr Deepak Bunger and Dr Mujtaba Khan are employees of Intas Pharmaceuticals Ltd.

\section{References}

1. Asthana S, Chauhan S and Labani S: Breast and cervical cancer risk in India: An update. Indian J Public Health 58: 5-10, 2014.

2. Harjani RR, Janaki MG, Somashekhar M, Ponni A, Alva RC, Koushik K, Kannan RA and Sathyamurthy A: Feasibility of Concurrent Chemoradiation in Cervical Cancer Patients From Rural Background. Clin Ovarian Other Gynecol Cancer 7: 29-32, 2014.

3. Nandakumar A, Ramnath T and Chaturvedi M: The magnitude of cancer cervix in India. Indian J Med Res 130: 219-221, 2009.

4. Bobdey S, Sathwara J, Jain A and Balasubramaniam G: Burden of cervical cancer and role of screening in India. Indian J Med Paediatr Oncol 37: 278-285, 2016.

5. Boussios S, Seraj E, Zarkavelis G, Petrakis D, Kollas A, Kafantari A, Assi A, Tatsi K, Pavlidis N and Pentheroudakis G: Management of patients with recurrent/advanced cervical cancer beyond first line platinum regimens: Where do we stand? A literature review. Crit Rev Oncol Hematol 108: 164-174, 2016.

6. Morris M, Eifel PJ, Lu J, Grigsby PW, Levenback C, Stevens RE, Rotman M, Gershenson DM and Mutch DG: Pelvic radiation with concurrent chemotherapy compared with pelvic and paraaortic radiation for high-risk cervical cancer. N Engl J Med 340: 1137-1143, 1999.

7. National Cancer Institute. NCI Issues Clinical Announcement on Cervical Cancer: Chemotherapy Plus Radiation Improves Survival. February 22.

8. Takekida S, Fujiwara K, Nagao S, Yamaguchi S, Yoshida N, Kitada F, Kigawa J, Terakawa N, Nishimura R and Phase II: Phase II study of combination chemotherapy with docetaxel and carboplatin for locally advanced or recurrent cervical cancer. Int J Gynecol Cancer 20: 1563-1568, 2010.

9. Chen CC, Wang L, Lin JC and Jan JS: The prognostic factors for locally advanced cervical cancer patients treated by intensity-modulated radiation therapy with concurrent chemotherapy. J Formos Med Assoc 114: 231-237, 2015.

10. Monk BJ, Alberts DS, Burger RA, Fanta PT, Hallum AV III, Hatch KD and Salmon SE: In vitro phase II comparison of the cytotoxicity of a novel platinum analog, nedaplatin (254-S), with that of cisplatin and carboplatin against fresh, human cervical cancers. Gynecol Oncol 71: 308-312, 1998. 
11. Naik R and Khan MA: Doceaqualip in a patient with prostate cancer who had an allergic reaction to conventional docetaxel: A case report. Mol Clin Oncol 6: 341-343, 2017.

12. Ahmad A, Sheikh S, Taran R, Srivastav SP, Prasad K, Rajappa SJ, Kumar V, Gopichand M, Paithankar M, Sharma M, et al: Therapeutic efficacy of a novel nanosomal docetaxel lipid suspension compared with taxotere in locally advanced or metastatic breast cancer patients. Clin Breast Cancer 14: 177-181, 2014.

13. Ashraf M SR, Khan MA, Shah M, Bhat Y and Wani ZA: Efficacy and safety of a novel nanosomal docetaxel lipid suspension (NDLS) as an anti cancer agent a retrospective study. Ann Oncol. 27 (suppl_9): mdw579.008, 2016.

14. Kong TW, Chang S-J, Paek J, Yoo S-C, Yoon J-H, Chang K-H, Chun $\mathrm{M}$ and Ryu H-S: Comparison of concurrent chemoradiation therapy with weekly cisplatin versus monthly fluorouracil plus cisplatin in FIGO stage IIB-IVA cervical cancer. J Gynecol Oncol 23: 235-241, 2012.

15. Nam EJ, Lee M, Yim GW, Kim JH, Kim S, Kim SW, Kim JW and Kim YT: Comparison of carboplatin- and cisplatin-based concurrent chemoradiotherapy in locally advanced cervical cancer patients with morbidity risks. Oncologist 18: 843-849, 2013.

16. Adams M, Kerby IJ, Rocker I, Evans A, Johansen K and Franks CR; The Swons Gynaecological Cancer Group: A comparison of the toxicity and efficacy of cisplatin and carboplatin in advanced ovarian cancer. Acta Oncol 28: 57-60, 1989.
17. Weiss GR, Green S, Hannigan EV, Boutselis JG, Surwit EA, Wallace DL and Alberts DS: A phase II trial of carboplatin for recurrent or metastatic squamous carcinoma of the uterine cervix: A Southwest Oncology Group study. Gynecol Oncol 39: 332-336, 1990.

18. Schwab CL, English DP, Roque DM and Santin AD: Taxanes: Their impact on gynecologic malignancy. Anticancer Drugs 25 522-535, 2014

19. Rein DT, Kurbacher CM, Breidenbach M, Schöndorf T, Schmidt T, König E, Göhring UJ, Blohmer JU and Mallmann P: Weekly carboplatin and docetaxel for locally advanced primary and recurrent cervical cancer: A phase I study. Gynecol Oncol 87: 98-103, 2002

20. Nagao S, Fujiwara K, Oda T, Ishikawa H, Koike H, Tanaka H and Kohno I: Combination chemotherapy of docetaxel and carboplatin in advanced or recurrent cervix cancer. A pilot study. Gynecol Oncol 96: 805-809, 2005

21. Lyman GH, Dale DC, Legg JC, Abella E, Morrow PK, Whittaker S and Crawford J: Assessing patients' risk of febrile neutropenia: Is there a correlation between physician-assessed risk and model-predicted risk? Cancer Med 4: 1153-1160, 2015. 